Western University

Scholarship@Western

\title{
$5-2020$
}

\section{A systematic review of occupational therapy interventions in the transition from homelessness}

Roxanne Isard Ms.

Western University, risard2@uwo.ca

Carrie Anne Marshall

Western University, carrie.marshall@uwo.ca

Leonie Boland

University of Plymouth, Plymouth, UK, leonie.boland@plymouth.ac.uk

Lee Ann Westover

Teachers College at Columbia University, leeannwestover@gmail.com

Sharon A. Gutman

Columbia University, sg2422@cumc.columbia.edu

Follow this and additional works at: https://ir.lib.uwo.ca/otpub

Part of the Occupational Therapy Commons

\section{Citation of this paper:}

Carrie Anne Marshall, Leonie Boland, Lee Ann Westover, Roxanne Isard \& Sharon A. Gutman (2021) A systematic review of occupational therapy interventions in the transition from homelessness,Scandinavian Journal of Occupational Therapy, 28:3, 171-187, DOI: 10.1080/ 


\section{Scandinavian Journal of Occupational Therapy}

\section{A systematic review of occupational therapy interventions in the transition from homelessness}

\section{Carrie Anne Marshall, Leonie Boland, Lee Ann Westover, Roxanne Isard \&}

Sharon A. Gutman

To cite this article: Carrie Anne Marshall, Leonie Boland, Lee Ann Westover, Roxanne Isard \& Sharon A. Gutman (2020): A systematic review of occupational therapy interventions in the transition from homelessness, Scandinavian Journal of Occupational Therapy

To link to this article: https://doi.org/10.1080/11038128.2020.1764094

View supplementary material 주

曲 Published online: 01 Jun 2020.

Submit your article to this journal

Q View related articles ¿

View Crossmark data 


\title{
A systematic review of occupational therapy interventions in the transition from homelessness
}

\author{
Carrie Anne Marshall ${ }^{a}$ D, Leonie Boland ${ }^{b}$ (D), Lee Ann Westover ${ }^{c}$, Roxanne Isard ${ }^{d}$ and Sharon A. Gutman ${ }^{e}$

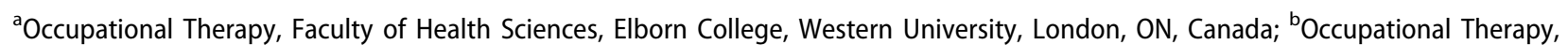 \\ Faculty of Health and Human Sciences, Peninsula Allied Health Centre, University of Plymouth, Plymouth, UK; ' ${ }^{\top}$ Teacher's College,

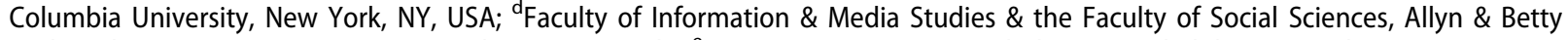

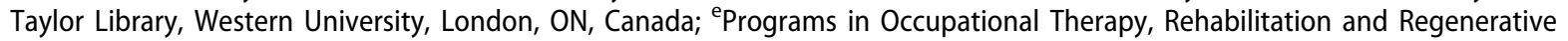 \\ Medicine, Columbia University, New York, NY, USA
}

\begin{abstract}
Background: Although systematic and scoping reviews have identified a range of interventions for persons experiencing homelessness, no known reviews have captured the range and quality of intervention studies aimed at supporting a transition from homelessness.

Objectives: To capture the range and quality of occupational therapy intervention studies aimed at supporting a transition to housing following homelessness.

Method: Using Joanna Briggs Institute (JBI) guidelines, we conducted a systematic review including a critical appraisal and narrative synthesis of experimental studies.

Results: Eleven studies were included. Critical appraisal scores ranged from 33.3 to 88.9 of a possible score of $100(\mathrm{Mdn}=62.5 ; \mathrm{IQR}=33.4)$. The majority of studies evaluated interventions for the development of life skills $(n=9 ; 81.8 \%)$, and all were conducted in the USA. Several of the included studies were exploratory evaluation and feasibility studies, and all were quasiexperimental in design. Only three studies (27.2\%) incorporated a control group. Intervention strategies included (1) integrated group and individual life skills interventions $(n=6)$; (2) groupbased life skills interventions $(n=3)$; and (3) psychosocial and consultative interventions $(n=2)$.

Conclusions: Research evaluating occupational therapy interventions aimed at supporting homeless individuals as they transition to housing is in an early stage of development.

Significance: Implications for research and practice are discussed.
\end{abstract}

\section{ARTICLE HISTORY}

Received 17 January 2020

Revised 14 April 2020

Accepted 29 April 2020

\section{KEYWORDS}

Homeless persons; activities of daily living; occupational therapy; meaningful engagement; systematic review

\section{Introduction}

Homelessness in high-income countries continues to grow despite elaborate efforts to address this serious and preventable social problem [1-4]. Acknowledging this growing international issue, occupational therapy scholarship in the area of homelessness has been growing rapidly in the past several years in an effort to address this social justice and human rights problem. In a previous paper, our team has declared homelessness as a 'disability rights issue' due to the high prevalence of physical, cognitive, and mental health conditions experienced by homeless individuals [5]. Many individuals experiencing homelessness in high-income countries live in conditions where they have limited access to basic needs such as nutritious food, clean water, and environments in which they can maintain physical and emotional safety. The
United Nations goals for sustainable development implore nations around the world to seek social justice by addressing issues of inequality and human rights violations [6]. Growth in the literature on homelessness within occupational therapy demonstrates the profession's commitment to social justice, and clear motivation to address inequality.

Research focussing on homelessness in occupational therapy has explored the occupational experiences of homeless persons [5] and occupation-based interventions in the support of people who are currently homeless [7]. Fewer studies, however, have explored these areas in relation to the transition from homelessness to being housed [8]. There is an important and promising role for occupational therapists in enabling formerly homeless individuals to sustain a tenancy, and to thrive in their housing following

CONTACT Carrie Anne Marshall carrie.marshall@uwo.ca O Occupational Therapy, Faculty of Health Sciences, Elborn College, Western University, London, ON, Canada

(4) Supplemental data for this article can be accessed here.

(C) 2020 Informa UK Limited, trading as Taylor \& Francis Group 
homelessness $[9,10]$. Despite this potential, there is a relative paucity of literature on this topic, specifically related to the development and evaluation of intervention approaches. This is problematic as it limits research and the practice of occupational therapists who regard themselves as evidence-informed professionals [11], and who have the potential to contribute their unique skills to improve the lives of individuals as they leave homelessness [9]. Supporting individuals to leave homelessness and integrate within their communities is an important way that occupational therapists can address social inequalities, and has been identified as a priority of occupational therapy practice in this context [12].

\section{Interventions addressing homelessness in high- income countries}

A variety of strategies have been developed and implemented to support the health and housing needs of homeless individuals. The most widely recognized approaches include Housing First (HF) [13] and Critical Time Intervention (CTI) [14]. HF is an approach which emphasizes the immediate provision of housing while supports for managing mental health and substance use challenges are offered in the form of case management or assertive community treatment [13]. CTI is an intervention designed for implementation during a 'critical time' in the life of a person who has lost their housing such as a hospital admission or shelter stay, and is composed of a threephase approach aimed at preventing on-going homelessness [15]. Although HF has been implemented and evaluated more broadly, both CTI and HF have a strong evidence base demonstrating effectiveness in reducing the number of days of homelessness $[14,16,17]$. Neither of these approaches, however, has consistently demonstrated effectiveness in addressing outcomes other than securing a tenancy. For instance, both have either poorly or inconsistently demonstrated effectiveness for community and social integration, reducing substance use, and amelioration of symptoms of mental illness following homelessness [18-21]. This has led scholars to question the extent to which these approaches can influence psychosocial outcomes, as such interventions have been designed primarily to enable homeless persons to secure a tenancy [14,18].

Securing a tenancy is critical to addressing the problem of homelessness in high-income countries [17]. Yet even when supported by existing approaches, those who have left homelessness frequently report feeling lonely and isolated [22], a lack of meaningful activity and boredom [9], on-going challenges with substance misuse [23] and poor community integration $[19,24]$. More is needed to enable thriving following homelessness for this vulnerable population. The focus of occupational therapy on optimal function and participation in a meaningful life means that the profession is optimally situated for developing and evaluating interventions targeting outcomes that are inadequately addressed by $\mathrm{HF}$ and CTI alone. Little is known, however, about the range and effectiveness of interventions developed by occupational therapy researchers to support a transition from homeless to housed.

\section{Occupational therapy interventions for supporting the transition to housing}

Reviews of occupational therapy approaches in the area of homelessness have been published in the existing literature. These include a systematic review of occupational therapy interventions for homeless persons published in 2011 [23], and a scoping review of occupation-based practices published in 2017 [2]. Both these studies primarily focus on strategies used during homelessness. Occupational therapists have a promising role in not only supporting individuals during homelessness but also as they transition to being housed $[8,9]$. Further, occupational therapy has the potential to improve existing interdisciplinary services by contributing an occupational perspective that may be used by a variety of professionals who support individuals as they leave homelessness. There are no known studies, however, exploring the range and quality of evidence for occupational therapy interventions designed to support homeless persons during this transition. There is a need both to aggregate and evaluate the quality of this literature to direct future research and practice efforts.

\section{The current study}

Occupational therapy is a promising approach for informing interventions that support thriving rather than simply sustaining a tenancy following homelessness. To support practice and to direct future research efforts, we designed this systematic review to aggregate and evaluate the quality of intervention studies by addressing the following research questions: (1) What interventions have been evaluated in existing literature that both include occupational therapists and are aimed at supporting homeless persons as they 
transition to being housed? and (2) What is the quality of existing literature on this topic?

\section{Material and methods}

We conducted a systematic review using Joanna Briggs Institute (JBI) guidelines [25] to capture existing literature evaluating occupational therapy interventions for supporting the transition from homeless to housed.

\section{Search strategy}

A search strategy was developed in collaboration with an Academic Research Librarian, an author on this study (RI), which was deployed in February 2019. Following Preferred Reporting Items for Systematic Reviews and Meta-Analyses (PRISMA) Guidelines [26], we searched six databases: EMBASE, CINAHL, PsychINFO, Medline, Nursing and Allied Health and Proquest Dissertations and Theses. We translated the search strategies using each database platform's command language, controlled vocabulary, and appropriate search fields using terms related to the concept of homelessness (e.g. homeless*, houseless), combined with terms pertaining to occupational therapy (e.g. occupation*) with a Boolean 'AND'. In addition to this search, we hand searched the reference lists of all included articles to identify any additional studies not captured using our search strategy. A sample of our Medline search is provided in Supplementary Appendix.

\section{Study selection}

Using two independent raters, we conducted a title and abstract search and full text review using Covidence, a cloud-based systematic review software programme [27]. At both the title and abstract, and full-text review stages, we included: (1) levels I and II evidence for effectiveness studies according to the JBI level of evidence hierarchy (experimental and quasiexperimental designs) [28]; (2) studies evaluating an intervention that involved an occupational therapist in its delivery; (3) studies involving persons with a history of homelessness within two years of study participation; (4) studies evaluating an intervention aimed at supporting a transition to housing following homelessness; (5) studies involving participants of all ages; and (6) all years and languages. We excluded articles that were (1) not subjected to peer review; (2) conference abstracts; (3) involved participants who were homeless due to fleeing war or conflict; (4) studies involving persons living in low-income countries. Any conflicts emerging at the title and abstract and full-text review phases were resolved through discussion and consensus. When we discovered that an article had reported on the same sample in more than one publication, we selected the article that reported on either a broader sample or over a longer intervention period. If an article was deemed to be a derivative publication (i.e. a secondary analysis of an existing sample), we selected the article with the earliest date of publication.

\section{Critical appraisal}

Two independent raters conducted a critical appraisal using the JBI Critical Appraisal Checklists for Randomized Controlled Trials and QuasiExperimental Studies [29]. We assigned a score of one to each criterion rated 'yes', and zero to items rated 'no'. When there was insufficient data in the study to determine a rating, we contacted the study authors to gather additional information to inform scoring. After rating each study independently, we compared our ratings and through discussion, arrived at a consensus score. We converted the scores on each rating form to a percentage score between 0 and 100 to facilitate comparison as the total criteria scored on each form differed based on the form used and study appraised. When a criterion was not applicable to the study that we were appraising, we calculated a percentage based on a reduced number of overall criteria. We did not exclude studies based on critical appraisal score to capture a full appreciation of the quality of existing literature on this topic in an effort to guide future research efforts. Studies were identified as 'low' quality if assigned a score of 0-24, 'low-moderate' if assigned a score of 25-49, 'moderate-high' if assigned a score of 50-74 and 'high' quality if assigned a score of 75-100.

\section{Data extraction}

Using Microsoft Excel (v.18.11), we developed a data extraction form to capture the following information for studies included in our review: study design; sample country; sample size, gender, age, race, sexual orientation, employment history, educational attainment, clinical characteristics; intervention description; and outcomes. Two independent raters extracted data using identical forms. Once data were extracted by each rater, we compared information entered, and 

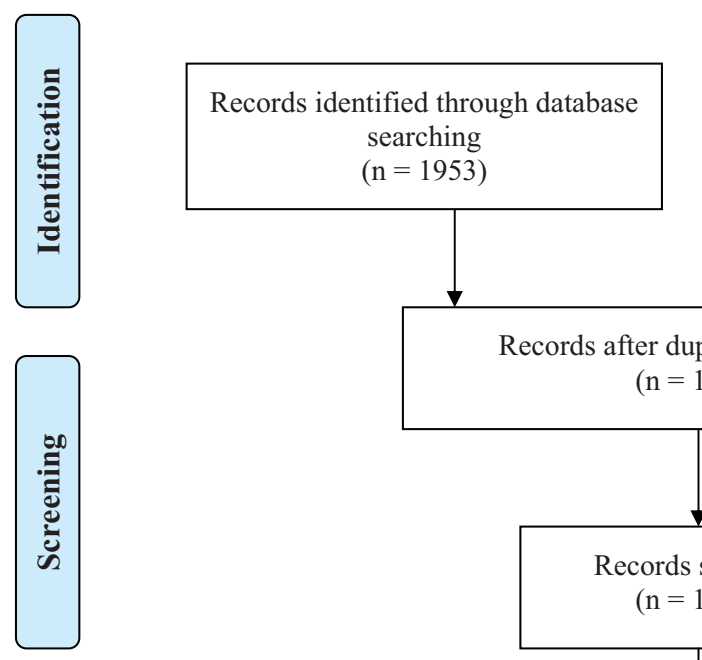

$(\mathrm{n}=1953)$
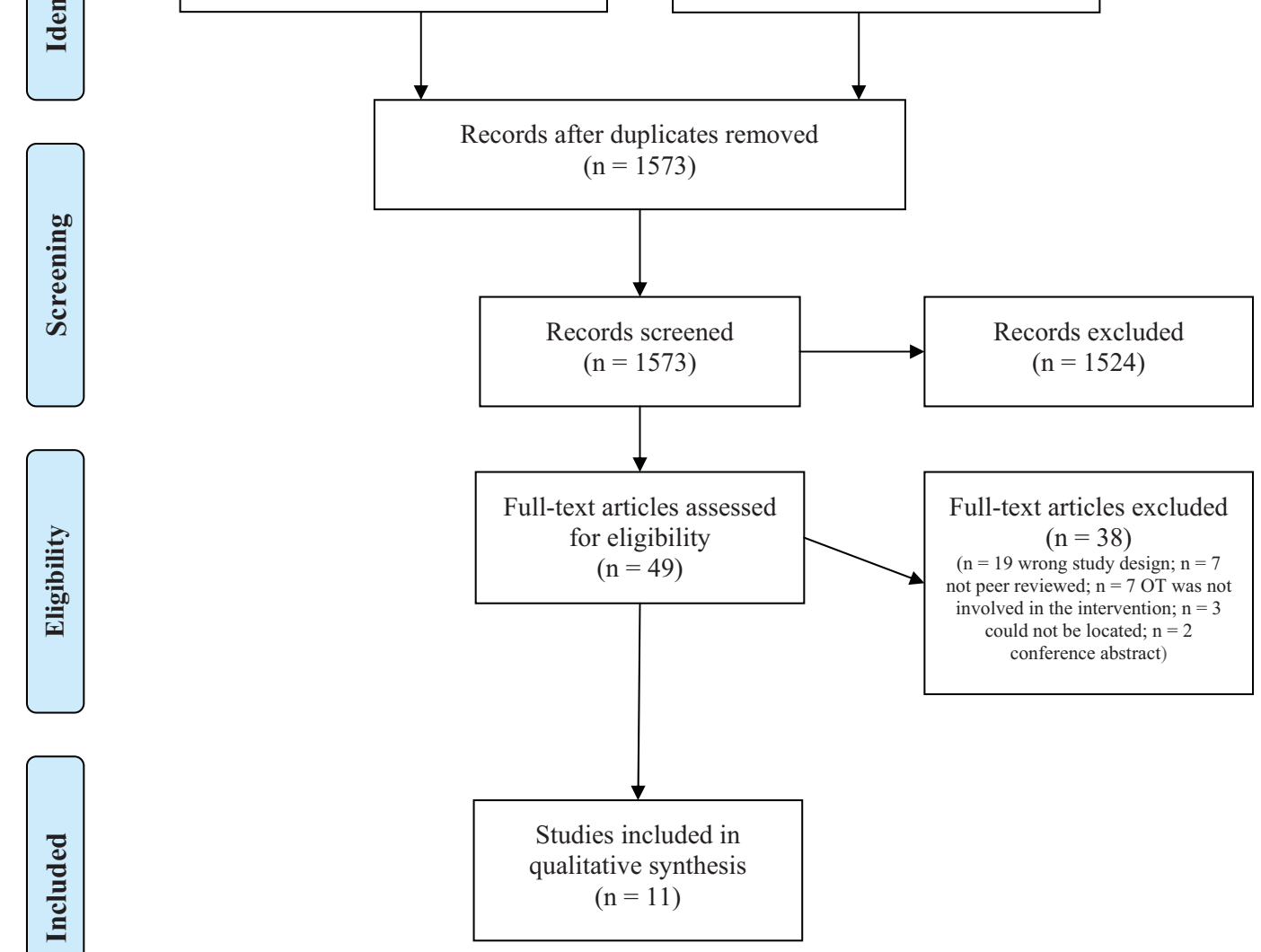

Figure 1. PRISMA flow diagram.

resolved any conflicts through discussion and consensus.

\section{Narrative synthesis}

The principal investigator categorized each article according to intervention approach and presented this categorization to two other members of the research team (L. B. and L. W.). This categorization of articles was refined through discussion and consensus. Articles grouped similarly were then described in detail in tables specific to the intervention approach. The findings of these studies were then synthesized in a narrative description, which is presented according to each intervention category in the results section of this paper.

\section{Results}

From a total of 1953 articles identified through our database and manual searches, a total of 11 studies were included in our review. See Figure 1 for our PRISMA flow diagram, which details our selection process.

\section{Study characteristics}

All of the studies included in this review were conducted in the USA between 2004 and 2018. Of 11 total studies, all were quasi-experimental in design.

\section{Participant characteristics}

Included studies represented a total of 443 participants, with samples ranging from 10 to 57 . Of these, 229 participants were identified as female (51.7\%), 204 as male $(46 \%)$, and the gender of 10 participants (2.3\%) was not specified. Race of participants included Black $(n=176,39.7 \%)$, White $(n=130$, 29.3\%), Hispanic $(n=19,4.3 \%)$, Indigenous $(n=3$, $0.6 \%)$, Other $(n=33,7.4 \%)$, and unspecified $(n=73$, $16.5 \%)$. None of the studies included in this review 
Table 1. Description of Included studies $(n=11)$.

\begin{tabular}{|c|c|}
\hline \multicolumn{2}{|l|}{ Characteristic } \\
\hline Participant characteristics & $n(\%)$ \\
\hline Sample size (baseline) & $443(100)$ \\
\hline \multicolumn{2}{|l|}{ Gender } \\
\hline Female & $229(51.7)$ \\
\hline Male & $204(46.0)$ \\
\hline Not specified & $10(2.3)$ \\
\hline \multicolumn{2}{|l|}{ Race } \\
\hline Black & $176(39.7)$ \\
\hline White & $130(29.3)$ \\
\hline Hispanic & $19(4.3)$ \\
\hline Indigenous & $3(0.6)$ \\
\hline Asian & $0(0)$ \\
\hline Mixed race & $9(2.0)$ \\
\hline Other & $33(7.4)$ \\
\hline Unspecified & $73(16.5)$ \\
\hline LGBTQ2 & Unreported in all studies \\
\hline \multicolumn{2}{|l|}{ Employment } \\
\hline Unemployed & $166(37.5)$ \\
\hline Employed & $10(2.3)$ \\
\hline Unspecified & $267(60.3)$ \\
\hline \multicolumn{2}{|l|}{ Education } \\
\hline$<$ Secondary school & $34(7.7)$ \\
\hline Secondary school diploma or equivalent & $133(30.0)$ \\
\hline Some college or university & $132(29.8)$ \\
\hline College or university completed & $19(4.3)$ \\
\hline Unspecified & $125(28.2)$ \\
\hline & $n$ studies (\%) \\
\hline \multicolumn{2}{|l|}{ Country of publication } \\
\hline USA & $11(100)$ \\
\hline
\end{tabular}

Percentage sums do not all equal 100 due to rounding.

involved those of Asian race. None identified the sexual orientation of participants. Employment status of participants was primarily unspecified $(n=267$, $60.3 \%)$, followed by unemployed $(n=166,37.5 \%)$, and employed in at least a part-time capacity $(n=10$, $2.3 \%)$. Almost one-third of the participants had a secondary school diploma or equivalent $(n=133,30.0 \%)$ or some college or university $(n=132,29.8)$, followed by less than secondary school $(n=34,7.7 \%)$, and college or university completed $(n=19,4.3 \%)$. Educational status of participants was not reported for 125 participants (28.2\%). See Table 1 for a summary of the characteristics of participants in the studies included in this review.

\section{Critical appraisal}

Critical appraisal scores ranged from 33.3 to 88.9 $(\mathrm{Mdn}=62.5, \mathrm{IQR}=33.4)$. Three included studies (27.3\%) represented evidence in the low-moderate quality range [30-32], five (45.5\%) in the moderate-high quality range [33-37], and three (27.3\%) in the high-quality range [38-40]. Only three $(27.3 \%)$ of the included studies incorporated a control group $[36,38,39]$. See Tables $2-4$ for a summary of scores assigned to individual studies.

\section{Narrative synthesis}

Given the emerging nature of this body of literature, the lack of control group conditions in included studies, and the heterogeneity of outcomes measured, we could not proceed with a meta-analysis. Instead, we have conducted a narrative synthesis of included studies. The majority of these studies evaluated interventions aimed at developing life skills $(n=9,81.8 \%)$, with some designed to be delivered in an integrated manner with both group and individual components $(n=6,54.5 \%)$, and others designed to be delivered primarily in group format $(n=3,27.3 \%)$. Psychosocial and consultative interventions $(n=2$, 18.2\%) included a peer support community [37] and an occupational therapy direct intervention and consultation service [38]. Each of these is discussed in detail below.

\section{Integrated group and individual life skills interventions}

A total of six studies (54.5\%) evaluated life skills interventions that included both group and individual components, and were designed for delivery in an integrated manner [30-35]. See Table 2 for a summary of the characteristics of studies included in this category.

Five of the studies included in this category were conducted by the same principal investigator (Helfrich) and all evaluated the same intervention. This intervention was first developed and reported in 2006 [32], and subsequently refined over time [30-32,34]. Using psychoeducation and experiential learning, this manualized intervention was informed by the Model of Human Occupation [41], and designed to improve residential stability among formerly homeless persons. While originally involving eight 60-min sessions (four group and four individual) [32], it was expanded to include 12 sessions (six group and six individual) in the most recent publication evaluating this approach [30]. Both group and individual sessions were facilitated by an occupational therapist. Group components consisted of sessions focussing on the development of skills in (1) Room and self-care; (2) Money management; (3) Nutrition/ food management; and (4) Safe community participation. These modules were offered on a rotating basis, and participants could complete them at any time in a 6-month period. Individual sessions were scheduled collaboratively between the occupational therapist and participant and were designed to reinforce what was learned in group sessions. 


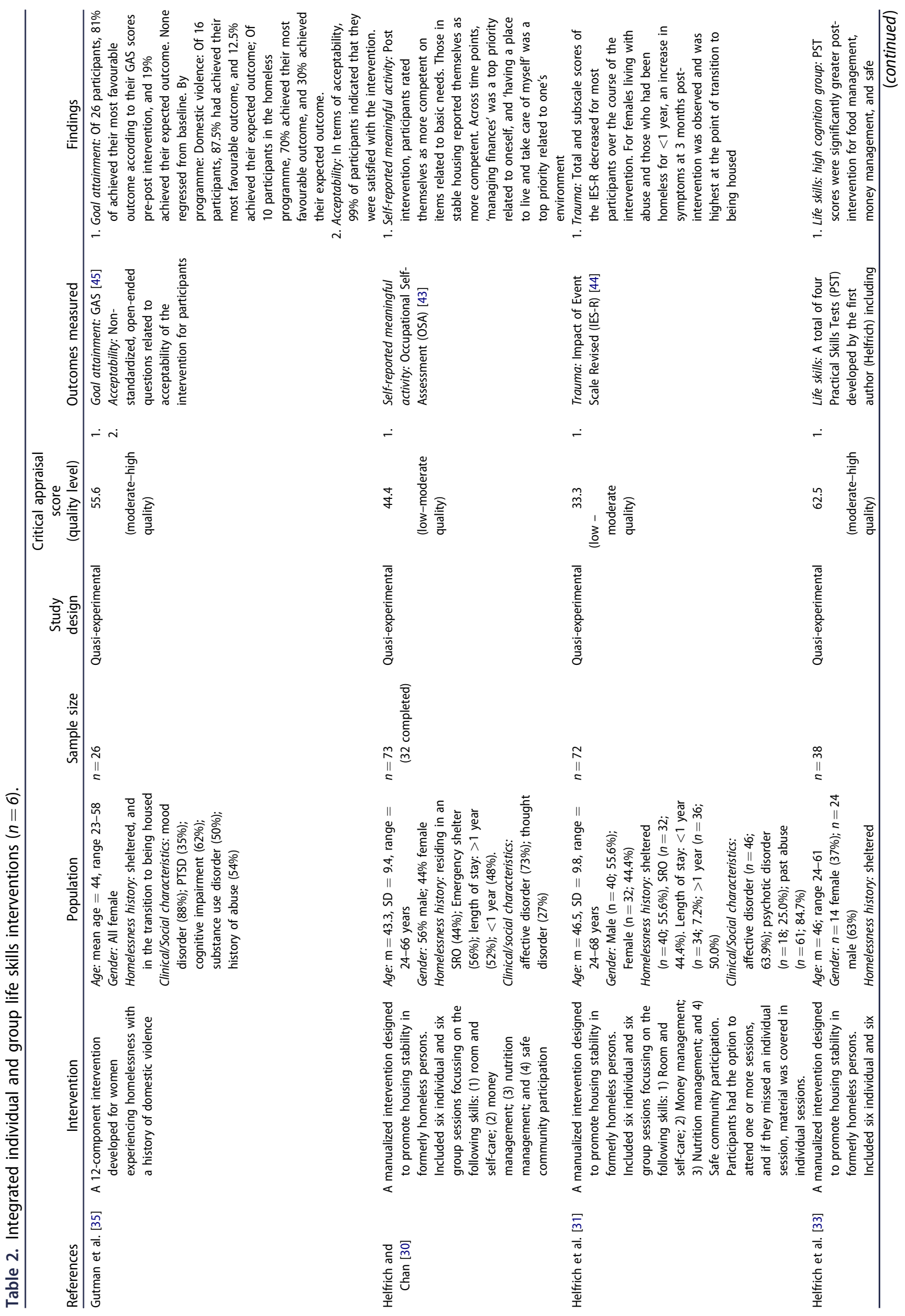




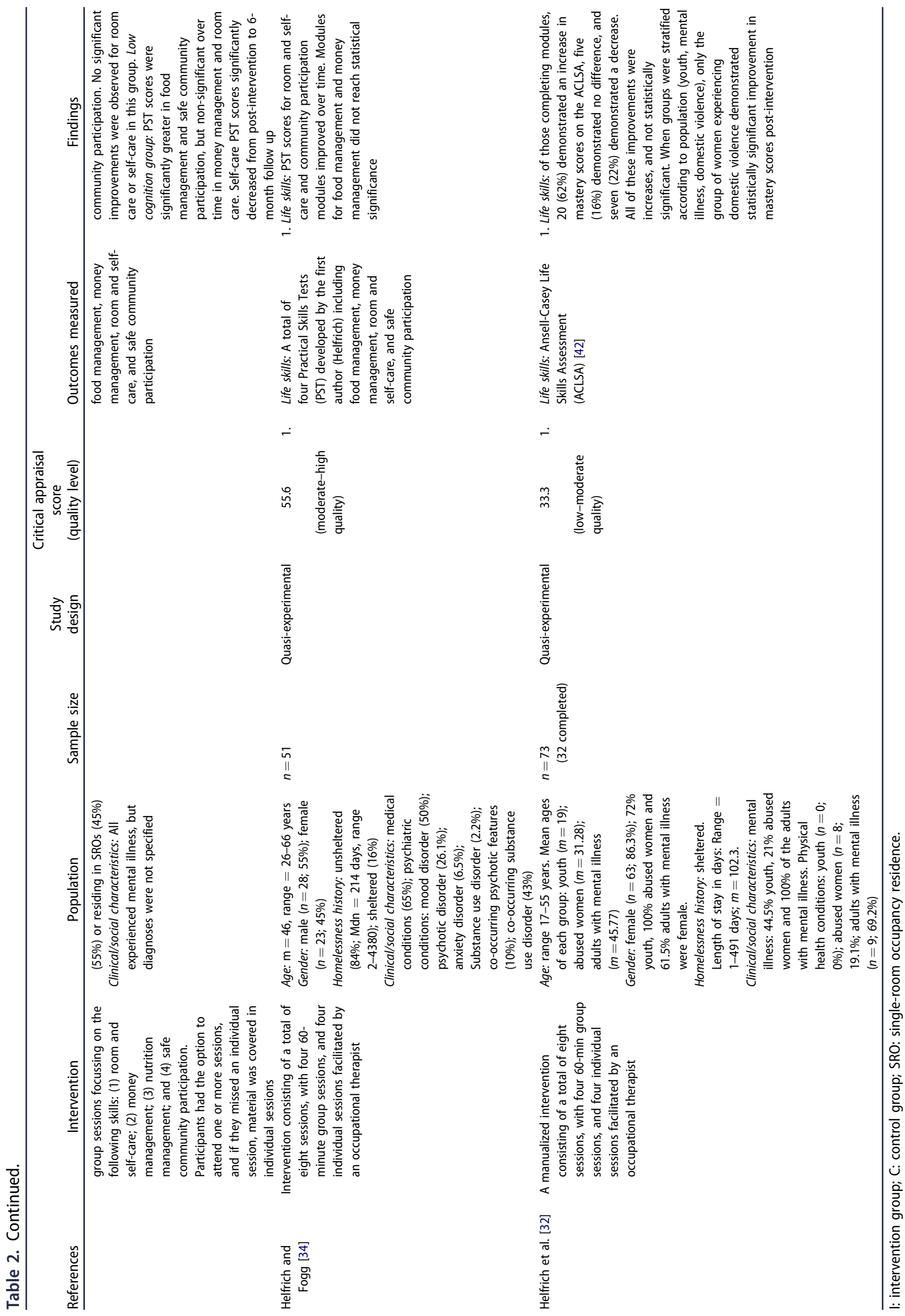


In three studies evaluating this intervention, the authors measured the impact of participation on life skills using the Ansell-Casey Life Skills Assessment (ACLSA) [42], and life skills knowledge using the Practical Skills Tests (PST). The latter measure (PST) was developed by the primary author of these studies (Helfrich) to evaluate this intervention [34]. In one study, the authors found that although there was a trend towards improvement in 'mastery of life skills' on the ACLSA, this relationship was non-significant for three groups of participants involved in the intervention (youth, adults with mental illness, and abused women) [32]. When 'life skills knowledge' was measured using the PST, participants in another study demonstrated significant improvement in knowledge of skills related to two modules (room and self-care, safe community participation). Improvement in knowledge of life skills related to the other modules (food management, money management) was not statistically significant [34]. The final study evaluated the impact of this intervention for groups of participants assessed as having either high or low cognition [33]. Post-intervention, participants in both groups demonstrated a significant increase only in knowledge of food management and safe community participation. Those in the high cognition group also demonstrated a significant increase in knowledge of money management. Room and self-care knowledge was not significantly different post-intervention for either group.

Two studies evaluated the impact of participating in this intervention on other outcomes including selfrated meaningful activities [30] using the Occupational Self-Assessment (OSA) [43], and trauma symptoms [31] using the Impact of Event ScaleRevised (IES-R) [44]. On the OSA, participants rated themselves as more competent on basic needs postintervention, with those who had sustained their housing rating themselves as more competent [34]. In determining the impact of this intervention on trauma symptoms, the study authors found that IES$\mathrm{R}$ scores decreased for most participants over the course of participating in the intervention which corresponded to an overall decrease in trauma symptoms [31].

A second integrated life-skills intervention with both group and individual sessions was designed for homeless women receiving support in a domestic violence programme [35]. The women were invited to attend group and individual sessions weekly for one hour over a 6-month period. This approach included 12 components, which were identified through a needs assessment conducted prior to the initiation of this study. Components included (1) safety planning; (2) drug and alcohol awareness; (3) safe sex practices; (4) assertiveness and advocacy skill training; (5) anger management; (6) stress management; (7) boundary establishment and limit setting; (8) vocational and educational skill training; (9) money management; (10) housing application; (11) leisure exploration; and (12) hygiene, medication routine, and nutrition. In group and individual sessions, women were encouraged to role play and receive feedback from other group members and the occupational therapist relating to real-life scenarios in their daily lives. The authors of this study used Goal Attainment Scaling (GAS) [45] to measure individualized goals identified by the women prior to participating in the intervention, as well as a measure of 'acceptability', including open-ended questions aimed at assessing participants' views of the approach. Following participation, the study authors identified that $81 \%$ of the women achieved their most favourable outcome on individualized GAS scores (i.e. goal achieved), and 19\% achieved their expected outcome (i.e. no change from baseline). In terms of acceptability, 99\% of participants identified that they were satisfied with the intervention.

\section{Group-based life skills intervention}

Three studies (27.3\%) evaluated the effectiveness of a group-based life skills intervention for homeless persons in the transition to being housed called Supporting Many to Achieve Residential Transition (SMART) $[36,39,40]$. See Table 3 for a summary of the characteristics of studies included in this category.

Authors of studies evaluating SMART describe this approach as an 'apartment living program' focussing on budgeting, cleaning and laundry, medication management, and negotiating conflict with roommates and neighbours [36]. One module was presented each week to participants in a group format augmented by DVD (video) as a facilitation tool. The intervention was delivered in two-hour sessions over a 6-week period including the following modules: (1) preparing for the housing interview and application process; (2) managing an apartment; (3) being a good tenant and neighbour; (4) living optimally in the community; (5) managing money; and (6) maintaining health and wellness. To maintain consistency across facilitators, the programme has been manualized and includes instructor resources, practice activities, and fidelity checklists [36]. 
SMART was evaluated in the studies included in this review on a variety of outcomes including individualized goal attainment using GAS [45], occupational performance using the Canadian Occupational Performance Measure (COPM) [46], social skills using the Interpersonal Skills Scale (ISS) [47], quality of life using the Manchester Short Assessment of Quality of Life Scale (MANSA) [48], housing status and community functioning, and satisfaction with the intervention. In evaluating the impact of SMART on GAS, participation was associated with significant improvements post-intervention in two studies $[39,40]$. Two studies identified significant improvements in quality of life, with MANSA scores increasing significantly post-intervention with large effect sizes: $(d=-1.34)$ [36]; and $(d=1.30)$ [39]. In one study, COPM performance and satisfaction scores were measured pre- and post-intervention, and were found to have significantly increased with large effect sizes on both subtests (performance: $d=-1.86$; satisfaction: $d=-1.85$ ) [36]. Social skills were also found to increase following participation in SMART as measured by the ISS, also with a large effect size $(d=-2.98)$ [36]. In one study, housing status and community functioning was assessed via interviews with SMART participants' case managers [40]. The authors reported that three months post-intervention, four of six participants receiving SMART had been housed, and one had secured employment. At 6months post-intervention, the same participants had sustained their housing for 3-5 months. Finally, two of the studies included in this review measured participant satisfaction with SMART [36,40]. Participants reported a high degree of satisfaction with the intervention, indicating on a 3-point Likert scale developed by the study authors that that they found the modules to be personally relevant, interesting and engaging, easy to understand, and provided sufficient opportunities for practice $[36,40]$.

\section{Psychosocial and consultative interventions}

Two studies (18.2\%) evaluated psychosocial and consultative interventions aimed at supporting homeless persons as they made the transition from homeless to housed. These included a peer support community [37] and an occupational therapy individual intervention and consultation service [38]. See Table 4 for a summary of the characteristics of studies included in this category.

The peer support community (PSC) intervention was facilitated with individuals living in an abstinence-based permanent supportive housing programme for individuals living with substance use disorders following homelessness [37]. The approach was led by an occupational therapist, who began by educating residents about peer support, and the value of a PSC. Following this, residents were invited to express interest in taking a peer leadership role. Group sessions aimed at developing leadership and communication skills were facilitated. The group was then supported to develop mission statements, and collaborate on rules of conduct, community purpose, and self-identified goals. After 10-weeks of supporting the community develop, the occupational therapist withdrew from the community to enable the PSC to function independently. The study authors measured quality of life using the Quality of Life Rating Scales (QOLR) [49], social support using the Medical Outcomes Study Social Support Survey (MOS-SSS) [50], motivation using the Volitional Questionnaire (VQ) [51], and frequency of substance use relapse. Significant improvements were observed in social support and substance use relapse post-intervention, with an increase in all three subtests of the MOS-SSS with moderate to large effect sizes (emotional/informational support: $r=0.628$; affectionate support: $r=0.493$; tangible support: $r=0.494$ ), and a significant reduction in the chance of substance use relapse to $7 \%$ from $24 \%$ measured in the year prior to implementing the PSC [37]. Quality of life and motivation scores were not statistically different postintervention.

The second study evaluated the impact of an occupational therapy direct intervention and consultation service on goal attainment and housing status [38]. The occupational therapist completed an initial assessment composed of a cognitive assessment and the COPM [46]. Following this, participants in the experimental condition were provided with on-going case management and both the participant and case manager were provided with access to an occupational therapist consultant. Regular meetings occurred between the occupational therapist and case managers, who on request, were provided with information about health conditions and helpful intervention strategies. The occupational therapist delivered weekly activity-based groups and provided information to case managers regarding the progress of participants in group sessions. Groups included diabetes education, life skills management, exercise, relaxation, crafts, gardening, and therapeutic horseback riding. The occupational therapist was also approached by the programme manager to provide group leadership skills training to case managers. Six-months post- 


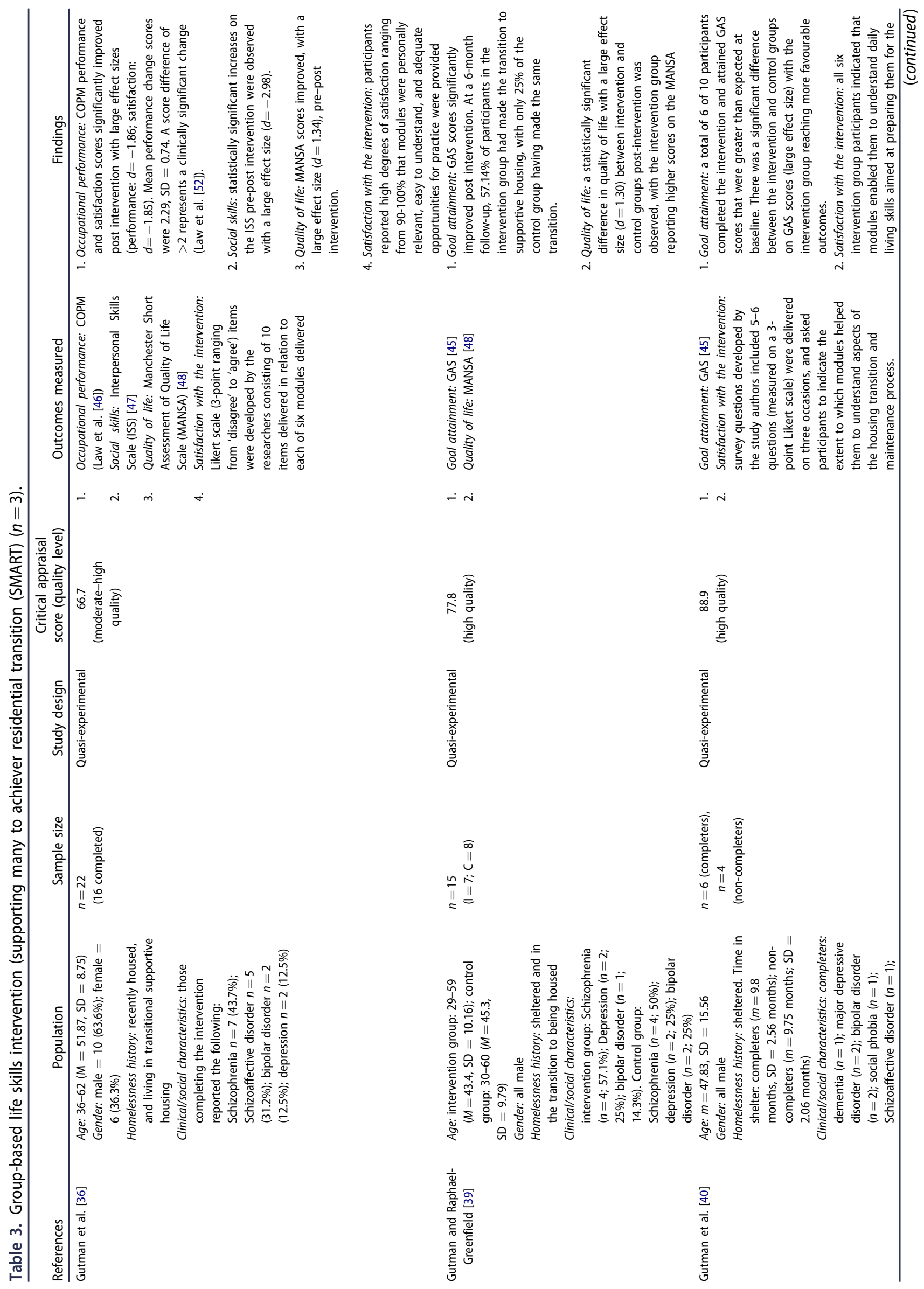




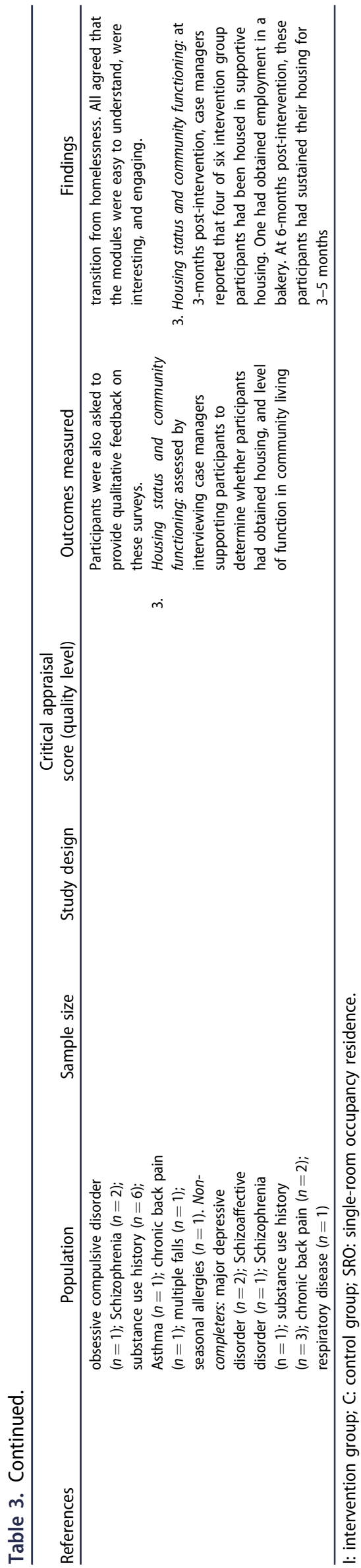

intervention, the study authors reported statistically significant gains in GAS scores for those in the experimental condition when compared with controls. After 1 year in the study, the housing status of those participating in this intervention improved over controls, but this relationship was non-significant [38].

\section{Discussion}

We conducted this study to identify the range and quality of literature evaluating occupational therapy interventions for supporting the transition to housing among individuals leaving homelessness. We identified a total of 11 experimental studies of moderate quality representing a total of 443 participants. All of the included studies were conducted in the USA and published between 2004 and 2018. All were quasiexperimental studies, and the majority were pre-post evaluation studies with no control group. Of those that included a control group, sample sizes were very small. Due to the lack of control group conditions and heterogeneity in study outcomes, we were unable to conduct a meta-analysis. Of the studies included, the vast majority $(81.8 \%)$ focussed on assisting homeless persons to develop life skills in the transition to housing. The recency, number of studies identified, and nature of the studies included in this review suggest that this body of literature is in a very early stage of development. To our knowledge, this is the only systematic review of occupational therapy interventions designed to support individuals as they transition from homeless to housed.

In conducting this review, we were surprised at the homogeneity of this body of literature. All of the included studies were conducted in the USA. Given that many studies in the occupational therapy literature on homelessness have been conducted in several high-income countries internationally $[5,7,53]$, we anticipated finding intervention studies conducted in a range of countries. Clearly, the findings of this review suggest otherwise. This is problematic, as the studies included in this review represent a narrow sociocultural context, and the findings of these studies cannot be reliably generalized to those living in countries outside of the USA. Those conducting research in other countries are encouraged to develop, implement, and evaluate interventions in their own unique contexts. Further, interventions identified in this review should be evaluated in countries other than the USA to determine whether outcomes are similar or different. This will improve the utility of these interventions in nations outside of the USA and help 


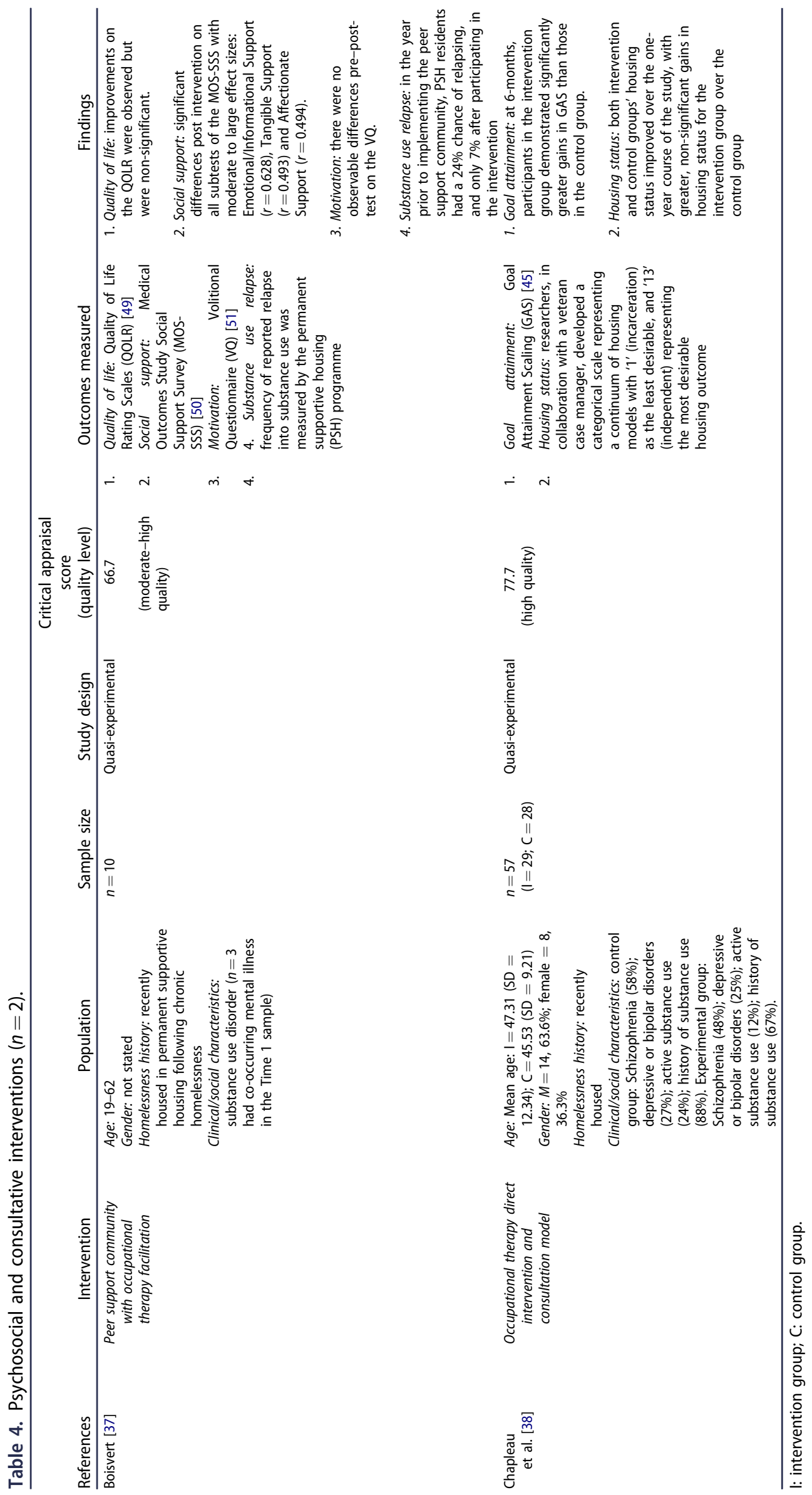


to inform necessary modifications needed to suit a range of sociocultural contexts.

Nearly all of the studies included in this review focussed on the development of life skills in the transition to being housed, and most were conducted by the same two principal investigators (Helfrich; Gutman). It makes sense that the focus of occupational therapy interventions might be dedicated to life skills given the profession's focus on supporting individuals to function optimally in activities in their daily lives despite health or social challenges [54]. This is an important focus in the support of homeless persons as individuals who have been homeless for long periods often report losing their ability to perform activities associated with being housed, and express concern about their ability to return to these activities upon securing a tenancy [55]. Life skills interventions, however, may not be the appropriate choice for some individuals who've experienced homelessness, as some participants in previous research report that they relearn independent living skills on their own in a relatively short period of time after securing a tenancy simply due to being in an environment in which performance of these skills becomes necessary [9]. What does persist as a problem for some is a lack of opportunities to participate in activities that are meaningful [9], resulting in profound boredom that has been associated with serious, negative impacts on mental well-being [56,57]. This is not to suggest that a focus on independent living skills is unimportant as many individuals who have experienced homelessness may have never learned these skills or may struggle to perform them due to the presence of a mental health or cognitive disorder. Instead, we intend to highlight that developing interventions to support formerly homeless persons to participate in activities that are meaningful is equally important and may support tenancy sustainment following homelessness. Existing interdisciplinary approaches, including HF and CTI have demonstrated poor or mixed findings for addressing key outcomes including community integration, substance use, and symptoms of mental illness $[18,21,58]$. There is a close relationship between engagement in meaningful activity and mental well-being, substance use and belonging for individuals experiencing homelessness [57], suggesting that employing strategies that address barriers to participation in meaningful activity may effectively target these outcomes.

Only two of the 11 studies included in this review incorporated a focus on engagement in meaningful activity [37,38]. In one of these studies, the intervention involved a range of activity-based groups in which those transitioning to housing could be involved [38]. Although housing status improved over time, and those participating in the intervention were more likely to experience improvements in their GAS scores than controls, other relevant psychosocial outcomes were not measured. Another intervention included in this review provided opportunities to develop a meaningful life role as a peer support leader in a programme for formerly homeless adults in substance use recovery [37]. In this study, the authors found that participating in the intervention was associated with improvements in social support and decreased substance use relapse, yet the sample size was very small $(n=10)$, and there was no control group condition. None of the studies included in this review measured meaningful activity engagement as an outcome of interest or attempted to identify any associations between this construct and other indices of psychosocial well-being. Overcoming barriers to inclusion in mainstream society, including the stigma of homelessness [59], is a complex problem for which an occupational therapy lens may be useful. Researchers and practitioners are encouraged to consider ways of incorporating approaches that help homeless persons engage in meaningful activity following homelessness as they develop and evaluate intervention approaches. Standardized measures of meaningful activity engagement (such as the Engagement in Meaningful Activities Survey [60]) and measures of boredom (such as the Multidimensional State Boredom Scale [61]) may be useful tools for evaluating the effectiveness of these strategies.

Life skills interventions identified in this review included those which integrated group and individual components [30-35], and a group-based intervention called SMART $[36,39,40]$. One of the integrated interventions was evaluated in five studies included in this review [30-34], and demonstrated mixed findings primarily for improving knowledge and confidence in independent living skills and also in the reduction of trauma symptoms [31]. Due to the lack of a control group, however, we can conclude little about whether outcomes identified were attributable to the intervention or to time and environmental factors alone. Although outcomes related to another integrated life skills intervention for homeless women receiving support in a domestic violence programme were encouraging [35], this study also lacked a control group. With respect to group-based interventions, the only intervention included in this category was SMART, 
which demonstrated more encouraging findings for a variety of key outcomes pertinent to supporting those leaving homelessness including goal attainment, performance and satisfaction in daily activities, social skills, quality of life, and housing and community functioning $[36,39,40]$. These studies all demonstrated impressive findings, and the incorporation of control conditions in two of these studies $[39,40]$ meant that outcomes could be more clearly attributed to the effects of the intervention. Although findings of these studies are promising, sample sizes were very small, and therefore, findings should be interpreted with caution.

One major gap in this body of literature was the lack of interventions aimed at engaging individuals leaving homelessness in employment. Given that the profession of occupational therapy and its scholarship are built on the underlying premise that human activities are inherently related to wellbeing [54], and employment is a substantial activity in which adults engage, the absence of employment-related interventions from this body of literature appears to be an oversight. Only one of the studies included in this review involved any component that was employment related [35]. This was a module delivered in the context of an integrated individual and group life skills intervention for women with histories of domestic violence and possible traumatic brain injury. The lack of attention to employment in interventions developed by occupational therapists for individuals making the transition to housing is puzzling given that being employed has the potential to both improve community and social integration, while simultaneously alleviating poverty. As poverty is a common cause of homelessness [2], improving one's income through employment is an important homelessness prevention strategy. There are a variety of interventions that have demonstrated effectiveness in the interdisciplinary literature for employment and other indices of psychosocial wellbeing among homeless persons including social enterprise (SE) $[62,63]$, individual placement and support (IPS) [64], and workrelated cognitive behavioural therapy (WCBT) [65]. Although the body of literature exploring employment interventions for homeless persons is relatively small [66], occupational therapy researchers have unique knowledge to contribute. Future occupational therapy research aimed at developing interventions to support homeless persons as they transition to housing may consider incorporating employment engagement strategies given the promise of employment in preventing on-going homelessness.

\section{Limitations}

There are several limitations of this study and the body of literature it represents. Many of the studies included in this review were primarily exploratory evaluation and feasibility studies meant to provide a foundation for future occupational therapy intervention research in the support of individuals leaving homelessness. Due to the nature of these studies, there are several methodological limitations that limit generalizability to the population of individuals leaving homelessness. Sample sizes were small, and only three of the included studies involved a control group. In the future, researchers are encouraged to evaluate interventions identified in this review, as well as other interventions using more robust research methods. We acknowledge that there are limitations to conducting research with homeless persons given inherent challenges associated with recruitment and retention in intervention studies with this population [67]. Researchers interested in conducting future intervention research with this population should be aware of these challenges and incorporate strategies to enhance retention and compensate for attrition including recruiting large baseline sample sizes and by incorporating intent-to-treat analyses. Future research should focus on increasing sample sizes, including control groups, and incorporating the use of randomized control trial designs where possible to improve both the reliability and validity of study findings. The fact that all of the included studies were conducted in the USA limits generalizability of the findings of this review to that context. Future intervention research should be conducted in other geographic contexts to improve generalizability. As the race of most participants included in this study was primarily Black and White, the findings of this review are limited to these races. Individuals representing races other than Black and White should be included in future occupational therapy research in this area. Similarly, the sexual orientation of participants included in this review was unspecified in all included studies. This is problematic, as individuals identifying as LGBTQ2+ are overrepresented in statistics on homelessness and sexual orientation is known to influence experiences of homelessness [68]. Sexual orientation may influence the relevance and effectiveness of interventions for this population, and we can conclude little about the effectiveness of interventions included in this review for this population. Finally, although our search was comprehensive and rigorous, we recognize that there is a possibility that may not have captured all available evidence on this topic. 


\section{Conclusion}

There is an important role for occupational therapy in designing and evaluating strategies to help individuals to thrive following homelessness. Existing interdisciplinary interventions have focussed primarily on tenancy sustainment for homeless persons. Although sustaining a tenancy is a critical outcome in the support of individuals who are leaving homelessness, there are opportunities to support individuals to thrive once housed. Improving one's ability to function effectively in the performance of life skills following homelessness, as well as addressing barriers to engagement in meaningful activity have the potential to promote thriving following homelessness. Few occupational therapy interventions, however, have been reported and evaluated in existing literature. We recommend that interventions developed and evaluated in future research include a balance of both developing life skills and engagement in meaningful activity. Researchers may consider incorporating measures of meaningful activity engagement in the evaluation of interventions and identify associations between this engagement and indices of psychosocial well-being. Where possible, future research designs should incorporate control groups and larger sample sizes to improve reliability and validity of study findings. Further, future research should be conducted in a range of sociocultural and geographic contexts.

\section{Disclosure statement}

The authors claim no financial interest or benefit arising from the direct applications of this research.

\section{ORCID}

Carrie Anne Marshall (D) http://orcid.org/0000-0002-

0592-7716

Leonie Boland (D) http://orcid.org/0000-0002-1476-324X

\section{References}

[1] Foundation Abbe Pierre - FEANTSA. Fourth overview of housing exclusion in Europe. 2019 [cited January 17 2019]; 161 pages. Available from: https:// www.feantsa.org/download/oheeu_2019_eng_web512 0646087993915253.pdf

[2] Gaetz S, Dej E, Richter T, et al. The state of homelessness in Canada. Toronto: Canadian Observatory on Homelessness Press; 2016 [cited 2019 Jan 17]. 85 pages. Available from: http://homelesshub.ca/sites/ default/files/SOHC16_final_20Oct2016.pdf

[3] National Alliance to End Homelessness. State of Homelessness Washington, DC, USA: NAEH; 2018 [cited 2019 Jan 17]. About 6 screens. Available from: https://endhomelessness.org/homelessness-in-america /homelessness-statistics/state-of-homelessness-report/

[4] Amore K. Severe housing deprivation in Aotearoa New Zealand: 2001-2013. 25 p. 2016 [2020 cited January 17]. Available from: http://www.healthyhousing.org.nz/wp-content/uploads/2016/08/Severehousing-deprivation-in-Aotearoa-2001-2013-1.pdf

[5] Marshall CA, Boland L, Westover LA, et al. Occupational experiences of homelessness: a systematic review and meta-aggregation. Scand J Occup Ther. 2019; [Nov 13, 14]. Available from:

[6] United Nations. Sustainable Development Goals. 2018. Available from: https://www.un.org/sustainabledevelopment/sustainable-development-goals/

[7] Roy L, Vallee C, Kirsh BH, et al. Occupation-based practices and homelessness: a scoping review. Can J Occup Ther. 2017;84:98-110.

[8] Marshall CA, Rosenberg MW. Occupation and the process of transition from homelessness. Can J Occup Ther. 2014;81:330-338.

[9] Marshall CA, Lysaght R, Krupa T. Occupational transition in the process of becoming housed following chronic homelessness. Can J Occup Ther. 2018; 85:33-45.

[10] Boland L. Transitioning from homelessness into a sustained tenancy: what enables successful tenancy sustainment? (The moving on project) [Ph.D.]. Ann Arbor: University of Plymouth (United Kingdom); 2018.

[11] Juckett LA, Robinson ML, Wengerd LR. Narrowing the gap: an implementation science research agenda for the occupational therapy profession. Am J Occup Ther. 2019;73:7305347010p1-7305347010p6.

[12] Marshall C, Gewurtz R, Barbic S, et al. Bridging the transition from homeless to housed: a social justice framework for guiding the practice of occupational therapists. 2020. Available from: https://bc79be03948b-49fb-a866-463bc7f2cc25.filesusr.com/ugd/fbaf23 _d369e5ec58f4427f9b50f43710b890f3.pdf

[13] Tsemberis S. Housing first: the pathways model to end homelessness for people with mental illness and addiction manual. Eur J Homelessness. 2011;5: 235-240.

[14] Herman DB, Conover S, Gorroochurn P, et al. Randomized trial of critical time intervention to prevent homelessness after hospital discharge. PS. 2011; 62:713-719.

[15] Herman D, Conover S, Felix A, et al. Critical Time Intervention: an empirically supported model for preventing homelessness in high risk groups. J Primary Prevent. 2007;28:295-312.

[16] Stergiopoulos V, Hwang SW, Gozdzik A, et al. Effect of scattered-site housing using rent supplements and intensive case management on housing stability among homeless adults with mental illness: a randomized trial. J Am Med Assoc. 2015;313: 905-915.

[17] Boland L, Slade A, Yarwood R, et al. Determinants of tenancy sustainment following homelessness: a systematic review. Am J Public Health. 2018;108: e1-e8. 
[18] Quilgars D, Pleace N. Housing first and social integration: a realistic aim? SI. 2016;4:5.

[19] Marshall C, Boland L, Westover L, et al. Interventions for community integration among homeless persons: a systematic review. Health Soc Care Community. 2019. DOI:10.1111/hsc.13030.

[20] Stergiopoulos V, O'Campo P, Hwang S, et al. At Home/Chez Soi Project: Toronto Site Final Report. Calgary, AB: Mental Health Commission of Canada. 36 p. 2014 [cited 2020 Jan 14]. Available from: https://www.mentalhealthcommission.ca/sites/default /files/At\%252520Home\%252520Report\%252520

Toronto\%252520ENG_0.pdf

[21] de Vet R, Beijersbergen MD, Jonker IE, et al. Critical time intervention for homeless people making the transition to community living: a randomized controlled trial. Am J Community Psychol. 2017;60:175-186.

[22] Bower M, Conroy E, Perz J. Australian homeless persons' experiences of social connectedness, isolation and loneliness. Health Soc Care Community. 2018;26:e241-e248.

[23] Somers JM, Moniruzzaman A, Palepu A. Changes in daily substance use among people experiencing homelessness and mental illness: 24-month outcomes following randomization to Housing First or usual care. Addiction. 2015;110:1605-1614.

[24] Henwood BF, Matejkowski J, Stefancic A, et al. Quality of life after housing first for adults with serious mental illness who have experienced chronic homelessness. Psychiatry Res. 2014;220:549-555.

[25] Aromataris E, Munn Z. Chapter 1: JBI systematic reviews. In: Aromataris E, Munn Z, editors. Joanna Briggs Institute Reviewer's manual. The Joanna Briggs Institute; 2017. Available from: https://reviewersmanual.joannabriggs.org/

[26] Moher D, Liberati A, Tetzlaff J, et al. Preferred reporting items for systematic reviews and metaanalyses: the PRISMA statement. Int J Surg. 2010;8: 336-341.

[27] VeritasHealthInnovation. Covidence systematic review software. Melbourne, Australia: Veritas Health Innovation; 2016.

[28] Munn Z, Porritt K, Aromataris E, et al. Supporting document for the joanna briggs institute levels of evidence and grades of recommendation. 19 p. 2014 [cited 2019 Dec 3]. Available from: https://joannabriggs.org/sites/default/files/2019-05/JBI\%20Levels\%20 of\%20Evidence\%20Supporting\%20Documents-v2.pdf

[29] Tufanaru C, Munn Z, Aromataris E, et al. Chapter 3: Systematic reviews of effectiveness. 2017 [cited 2019 Dec 3]. Available from: https://reviewersmanual.joannabriggs.org/

[30] Helfrich CA, Chan DV. Changes in self-identified priorities, competencies, and values of recently homeless adults with psychiatric disabilities. Am J Psychiatr Rehabil. 2013;16:22-49.

[31] Helfrich CA, Peters CY, Chan DV. Trauma symptoms of individuals with mental illness at risk for homelessness participating in a life skills intervention. Occup Ther Int. 2011;18:115-123.
[32] Helfrich CA, Aviles AM, Badiani C, et al. Life skill interventions with homeless youth, domestic violence victims and adults with mental illness. Occup Ther Health Care. 2006;20:189-207.

[33] Helfrich CA, Chan DV, Sabol P. Cognitive predictors of life skill intervention outcomes for adults with mental illness at risk for homelessness. Am J Occup Ther: Off Publ Am Occup Ther Assoc. 2011; 65:277-286.

[34] Helfrich CA, Fogg LF. Outcomes of a life skills intervention for homeless adults with mental illness. J Primary Prevent. 2007;28:313-326.

[35] Gutman SA, Diamond H, Holness-Parchment SE, et al. Enhancing independence in women experiencing domestic violence and possible brain injury. Occup Ther Ment Heal. 2004;20:49-79.

[36] Gutman SA, Raphael-Greenfield EI, Berg J, et al. Feasibility and satisfaction of an apartment living program for homeless adults with mental illness and substance use disorder. Psychiatry. 2018;81:228-239.

[37] Boisvert RA, Martin LM, Grosek M, et al. Effectiveness of a peer-support community in addiction recovery: participation as intervention. Occup Ther Int. 2008;15:205-220.

[38] Chapleau A, Seroczynski AD, Meyers S, et al. The effectiveness of a consultation model in community mental health. Occup Ther Ment Heal. 2012;28: 379-395.

[39] Gutman SA, Raphael-Greenfield EI. Effectiveness of a supportive housing program for homeless adults with mental illness and substance use: a two-group controlled trial. Br J Occup Ther. 2017;80:286-293.

[40] Gutman SA, Raphael-Greenfield EI, Simon PM. Feasibility and acceptability of a pilot housing transition program for homeless adults with mental illness and substance use. Occup Ther Health Care. 2016; 30:124-138.

[41] Kielhofner G. A model of human occupation: theory and application. 3rd ed. Baltimore: Lippincott Williams \& Wilkins; 2002.

[42] Nollan KA, Horn M, Downs AC, et al. Ansell-Casey Life Skills Assessment (ACLSA) and life skills guidebook manual. Seattle (WA): Casey Family Programs; 2002.

[43] Baron K, Kielhofner G, Lyenger A, et al. Occupational self assessment (OSA). Chicago: University of Illinois; 2006.

[44] Weiss D. The impact of event scale: revised. Boston: Springer; 2007.

[45] Kiresuk TJ, Sherman RE. Goal attainment scaling: a general method for evaluating comprehensive community mental health programs. Community Ment Health J. 1968;4:443-453.

[46] Law MC, Baptiste S, Carswell A, et al. Canadian Occupational Performance Measure (COPM) (4th ed.). Ottawa (ON): CAOT Publ. ACE; 2005.

[47] Schindler V. Occupational therapy in forensic psychiatry: role development and schizophrenia. Occup Ther Ment Heal. 2004;20:1-165.

[48] Priebe S, Huxley P, Knight S, et al. Application and results of the Manchester Short Assessment of 
Quality of Life (MANSA). Int J Soc Psychiatry. 1999;45:7-12.

[49] Gust T. Quality of Life Rating. Unpublished instrument; 1982.

[50] Sherbourne CD, Stewart AL. The MOS social support survey. Soc Sci Med. 1991;32:705-714.

[51] De las Heras CG, Geist R, Kielhofner G, et al. The Volitional Questionnaire (VQ) (Version 4.1). Chicago: University of Illinois; 2007.

[52] Law M, Polatajko H, Pollock N, et al. Pilot testing of the Canadian Occupational Performance Measure: clinical and measurement issues. Can J Occup Ther. 1994;61(4):191-197.

[53] Thomas Y, Gray M, McGinty S. A systematic review of occupational therapy interventions with homeless people. Occup Ther Health Care. 2011;25:38-53.

[54] Townsend E, Polatajko H. Enabling Occupation II: advancing an occupational therapy vision for health, well-being \& justice through occupation. 2nd ed. Ottawa: CAOT Press; 2013.

[55] Marshall CA, Lysaght R, Krupa T. The experience of occupational engagement of chronically homeless persons in a mid-sized urban context. J Occup Sci. 2017;24:165-180.

[56] Marshall CA, Roy L, Becker A, et al. Boredom and homelessness: a scoping review. J Occup Sci. 2019; 27:1-18.

[57] Marshall CA, Davidson L, Li A, et al. Boredom and meaningful activity in adults experiencing homelessness: a mixed-methods study. Can J Occup Ther. 2019;86:000841741983340-000841741983370.

[58] O’Campo P, Stergiopoulos V, Nir P, et al. How did a Housing First intervention improve health and social outcomes among homeless adults with mental illness in Toronto? Two-year outcomes from a randomised trial. BMJ Open. 2016;6:e010581.
[59] Belcher JR, DeForge BR. Social stigma and homelessness: the limits of social change. J Hum Behav Soc Environ. 2012;22:929-946.

[60] Goldberg B, Brintnell ES, Goldberg JT. The relationship between engagement in meaningful activities and quality of life in persons disabled by mental illness. Occup Ther Ment Heal. 2002;18:17-44.

[61] Ferguson KM, Bender K, Thompson SJ, et al. Employment status and income generation among homeless young adults: results from a five-city, mixed-methods study. Youth Soc. 2012;44:385-407.

[62] Fahlman SA, Mercer-Lynn KB, Flora DB, et al. Development and validation of the multidimensional state boredom scale. Assessment. 2013;20:68-85.

[63] Ferguson KM, Xie B. Feasibility study of the social enterprise intervention with homeless youth. Res Soc Work Pract. 2008;18:5-19.

[64] Poremski D, Rabouin D, Latimer E. A randomised controlled trial of evidence based supported employment for people who have recently been homeless and have a mental illness. Adm Policy Ment Health. 2017;44:217-224.

[65] Himle JA, Bybee D, Steinberger E, et al. Workrelated CBT versus vocational services as usual for unemployed persons with social anxiety disorder: a randomized controlled pilot trial. Behav Res Ther. 2014;63:169-176.

[66] Marshall C, Boland L, Westover L, et al. Employment interventions for homeless persons: a systematic review; 2020. [under review].

[67] Marshall C, Koop M, Keogh-Lim D, et al. Meaningful activity and boredom in the transition from homeless to housed: two case narratives. Can J Occup Ther. 2020. [Accepted].

[68] Ecker J, Aubry T, Sylvestre J. A review of the literature on LGBTQ adults who experience homelessness. J Homosex. 2019;66:297-323. 